\title{
Magnetic behavior of NiCu nanowire arrays: Compositional, geometry and temperature dependence
}

\author{
E. M. Palmero, ${ }^{1, a)}$ C. Bran, ${ }^{1}$ R. P. del Real, ${ }^{1}$ C. Magén, ${ }^{2}$ and M. Vázquez ${ }^{1}$ \\ ${ }_{1}^{1}$ Institute of Materials Science of Madrid, CSIC, Madrid 28049, Spain \\ ${ }^{2}$ Advanced Microscopy Laboratory (LMA), Institute of Nanoscience of Aragón (INA)-ARAID \\ and Department of Condensed Matter Physics, University of Zaragoza, Zaragoza 50018, Spain
}

(Received 23 April 2014; accepted 4 July 2014; published online 16 July 2014)

\begin{abstract}
Arrays of $\mathrm{Ni}_{100-\mathrm{x}} \mathrm{Cu}_{\mathrm{x}}$ nanowires ranging in composition $0 \leq \mathrm{x} \leq 75$, diameter from 35 to $80 \mathrm{~nm}$, and length from $150 \mathrm{~nm}$ to $28 \mu \mathrm{m}$ have been fabricated by electrochemical co-deposition of $\mathrm{Ni}$ and $\mathrm{Cu}$ into self-ordered anodic aluminum oxide membranes. As determined by X-ray diffraction and Transmission Electron Microscopy, the crystalline structure shows fcc cubic symmetry with [111] preferred texture and preferential $\mathrm{Ni}$ or $\mathrm{Cu}$ lattice depending on the composition. Their magnetic properties such as coercivity and squareness have been determined as a function of composition and geometry in a Vibrating Sample Magnetometer in the temperature range from 10 to $290 \mathrm{~K}$ for applied magnetic fields parallel and perpendicular to the nanowires axis. Addition of $\mathrm{Cu}$ into the $\mathrm{NiCu}$ alloy up to $50 \%$ enhances both parallel coercivity and squareness. For the higher $\mathrm{Cu}$ content, these properties decrease and the magnetization easy axis becomes oriented perpendicular to the wires. In addition, coercivity and squareness increase by decreasing the diameter of nanowires which is ascribed to the increase of shape anisotropy. The temperature dependent measurements reflect a complex behavior of the magnetic anisotropy as a result of energy contributions with different evolution with temperature. (C) 2014 AIP Publishing LLC.
\end{abstract}

[http://dx.doi.org/10.1063/1.4890358]

\section{INTRODUCTION}

Ordered arrays of magnetic nanowires exhibit specific properties compared to their bulk counterpart which make them technologically relevant for high-density recording, microelectronics, and sensing devices. ${ }^{1-3}$ In addition, they are considered as nearly ideal systems for fundamental studies on the magnetic and transport properties in dimensionally confined magnetic systems. ${ }^{4}$ Techniques making use of template assisted growth of magnetic nanostructures enable the fabrication of long range ordered nanosystems. Particularly, the electrochemical route has been proved to be very useful to prepare first anodic aluminium oxide (AAO) templates with highly ordered self-assembled nanopores by two-step anodization process, ${ }^{5,6}$ which are later filled by controlled electrodeposition of metallic materials. ${ }^{7-9}$

A number of studies on ferromagnetic/non-magnetic multilayered and binary nanowire arrays, including $\mathrm{CoCu}$, $\mathrm{FePd}, \mathrm{CoAg}, \mathrm{CoPd}$, and $\mathrm{NiCu},{ }^{10-13}$ have been reported in the literature due to their interesting magnetic and magnetotransport properties. The $\mathrm{NiCu}$ binary system has been previously investigated using different methods, but focusing on bulk materials and multilayered nanowires. ${ }^{14-18}$ Only few works are properly dealing with the magnetic behavior of $\mathrm{NiCu}$ alloy nanowires. ${ }^{1,19}$ On one hand, it is well established that individual Ni nanowires present easy axis along the wire axis as a consequence of its comparatively larger shape magnetic anisotropy energy. ${ }^{20,21}$ However, the magnetostatic interactions in densely packed nanowire arrays can modify

${ }^{\text {a)} E-m a i l: ~ e p a l m e r o @ i c m m . c s i c . e s ~}$ significantly the magnetic properties of the array when the magnetic field is applied in a configuration parallel to the nanowires. ${ }^{2}$ For that reason, it is interesting to grow nanowires using an alloy of a non-magnetic material and a ferromagnetic material. ${ }^{22}$ Adding non-magnetic elements (such as $\mathrm{B},{ }^{23} \mathrm{Cu},{ }^{24} \mathrm{Zn},{ }^{25}$ and $\mathrm{P}^{26}$ ) to magnetic nanowires is a good method to tailor their magnetic properties. On the other hand, this binary alloy has interesting properties, such as corrosion and wear resistance, high strength, and good giant magnetoresistance. , $^{, 13}$

In this work, we report a phenomenological investigation as function of temperature, on the magnetic behavior of $\mathrm{Ni}_{100-\mathrm{x}} \mathrm{Cu}_{\mathrm{x}}$ nanowires depending on the alloy composition, and the nanowires geometry characteristics, such as diameter and length.

\section{MATERIALS AND METHODS}

Highly ordered AAO membranes were prepared by means of a two-step anodization process from high purity $(99.999 \%)$ aluminium foils. The foils were ultrasonically degreased first in acetone for 10 min followed by another 10 $\min$ in ethanol. Then, they were electropolished in a mixture of $\mathrm{HClO}_{4}: \mathrm{C}_{2} \mathrm{H}_{5} \mathrm{OH}=1: 3(\mathrm{~V} / \mathrm{V})$ under a constant potential of $20 \mathrm{~V}$ for $2.5 \mathrm{~min}$. The electropolished aluminium disks were anodized in $0.3 \mathrm{M}$ oxalic acid solution at $40 \mathrm{~V}$ and $4{ }^{\circ} \mathrm{C}$ during $24 \mathrm{~h}$. The resulting alumina layer was chemically removed using a mixed solution of $\mathrm{CrO}_{3}$ and $\mathrm{H}_{3} \mathrm{PO}_{4}$. The surface was re-anodized under the same potential and temperature as in the first anodization, for $20 \mathrm{~h}$. After that, the remaining aluminium substrate was removed by a mixture solution of $\mathrm{CuCl}_{2} \cdot 2 \mathrm{H}_{2} \mathrm{O}$ and $\mathrm{HCl}$. Finally, the alumina 

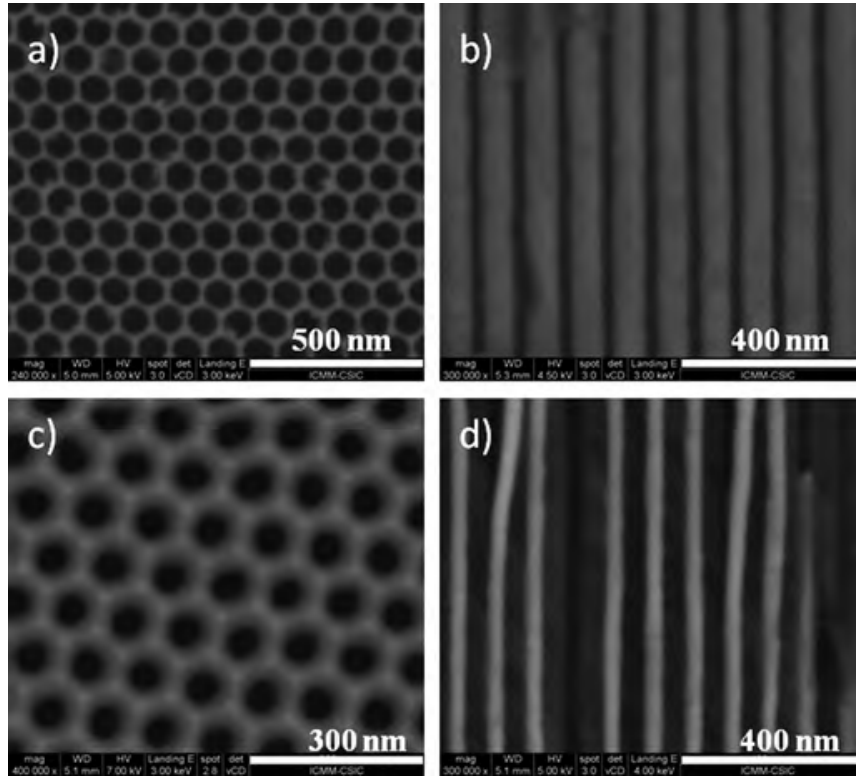

FIG. 1. Top view SEM images of an AAO membrane with pore diameter of $80 \mathrm{~nm}$ (a) and $35 \mathrm{~nm}$ (c), and the cross section of $80 \mathrm{~nm}$ diameter nanowires (b), and $35 \mathrm{~nm}$ diameter nanowires (d).

barrier layer was removed and the pores were widened by controlled chemical etching with a $\mathrm{H}_{3} \mathrm{PO}_{4}$ solution (5 wt. \%). A thin Au layer was sputtered at the bottom of the pores to serve as an electrode for electroplating the nanowires.

The electrochemical deposition was carried out in a three-electrode cell under constant potential at room temperature. The potential used in the electrodeposition was $-1.0 \mathrm{~V}$ vs. $\mathrm{Ag} / \mathrm{AgCl}$ reference electrode. Watts-type baths containing different amount of $\mathrm{NiSO}_{4} \cdot 6 \mathrm{H}_{2} \mathrm{O}(0.35 \mathrm{M}), \mathrm{CuSO}_{4} \cdot 5 \mathrm{H}_{2} \mathrm{O}$ $(0-0.06 \mathrm{M})$ and $\mathrm{H}_{3} \mathrm{BO}_{3}(0.30 \mathrm{M})$ were used in order to obtain $\mathrm{Ni}_{100-\mathrm{x}} \mathrm{Cu}_{\mathrm{x}}(0 \leq \mathrm{x} \leq 75)$ nanowires. The $\mathrm{pH}$ of the electrolytes was adjusted to 2.5 using diluted sulphuric acid. By controlling the time of the electrodeposition, nanowires with different lengths were grown.
A Nova Nano 230 High Resolution Scanning Electron Microscope (SEM) was used to characterize the structure of the membranes and the nanowires. The chemical composition of the nanowires was determined using an Energy Dispersive X-ray Spectrometer (EDS). The crystal orientation was established by means of an X-ray Diffractometer (XRD) on a PANalytical $X^{\prime}$ pert Pro in Bragg-Brentano geometry. Morphology and microstructure of the nanowires were analysed by High Resolution Transmission Electron Microscopy (HRTEM) and Selected Area Electron Diffraction (SAED) was carried out in an image-corrected FEI Titan Cube 60-300 operated at $300 \mathrm{kV}$, equipped with a CEOS aberration corrector for the objective lens to provide a point resolution below $1 \AA$. The magnetic measurements at room temperature were carried out using a Vibrating Sample Magnetometer (VSM), ADE system EV7 KLA-Tencor (maximum magnetic field of $\pm 1.8 \mathrm{~T}$ ) and at the temperature range of 10-290 K using a MLVSM 9T Oxford Instrument (maximum magnetic field of $\pm 9.0 \mathrm{~T}$ ). The magnetic field was applied parallel and perpendicular to the nanowires.

\section{RESULTS AND DISCUSSION}

SEM measurements have allowed us to determine the pore diameter which is tuned between 35 and $80 \mathrm{~nm}$, as well as the interpore distance of $105 \mathrm{~nm}$. The thickness of the membranes is $40 \mu \mathrm{m}$ and the length of the nanowires varies between $150 \mathrm{~nm}$ and $28 \mu \mathrm{m}$. Figure 1 shows the top and cross sectional view of selected arrays of 35 and $80 \mathrm{~nm}$ in diameter and $8 \mu \mathrm{m}$ long $\mathrm{Ni}_{55} \mathrm{Cu}_{45}$ nanowires embedded into $\mathrm{AAO}$ template.

The XRD spectra of the series of $\mathrm{Ni}_{100-\mathrm{x}} \mathrm{Cu}_{\mathrm{x}}$ nanowire arrays with different compositions embedded into AAO templates are shown in Figure 2. The diffraction patterns show that the Ni nanowires crystallize in face-centered cubic $(f c c)$ structure with a [220] preferred orientation (Figure 2(a)). $\mathrm{NiCu}$ nanowires crystallize in $f c c$ structure mainly along the [111] direction but showing also peaks in [200], [220], and

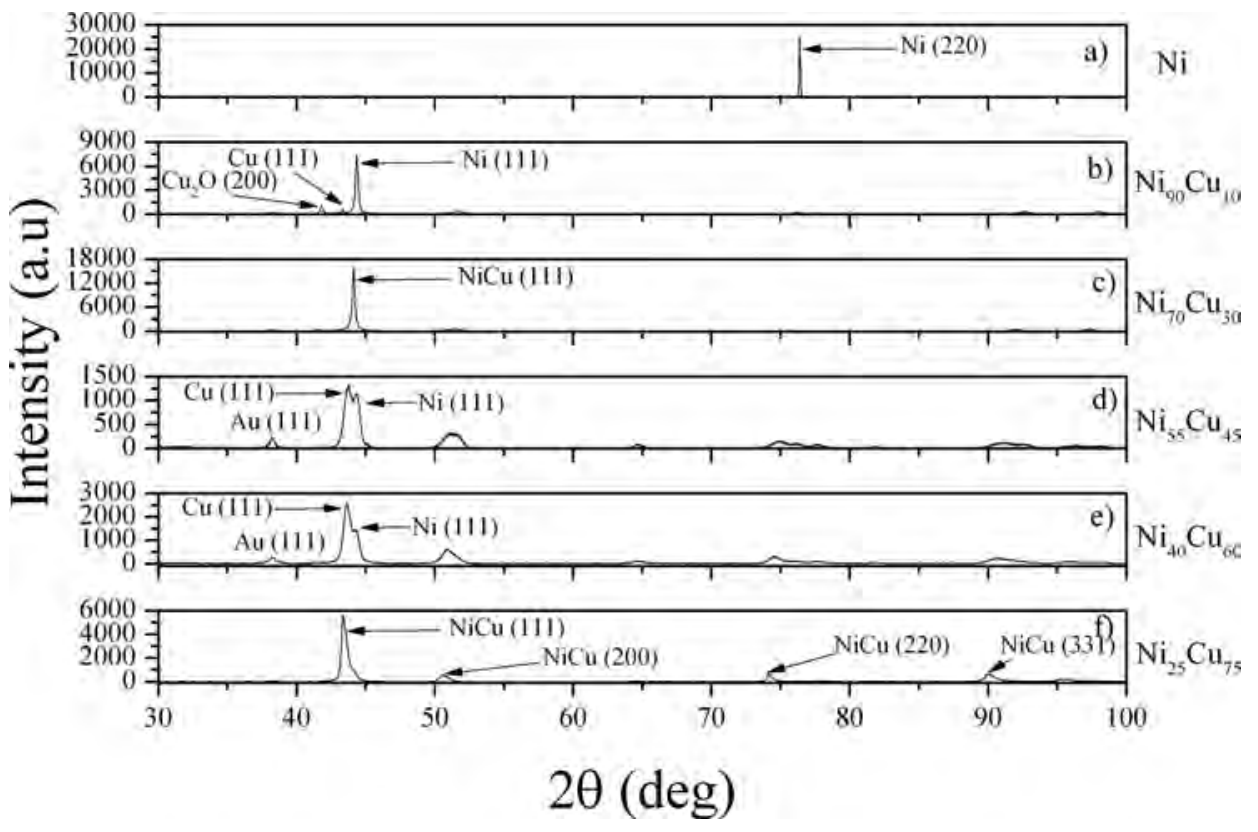

FIG. 2. XRD spectra of $\mathrm{NiCu}$ nanowires with different composition. 
[311]. For small $\mathrm{Cu}$ content (e.g., $\mathrm{x}=10$ in Figure 2(b)), two different [111] $f c c$ phases for $\mathrm{Ni}$ and $\mathrm{Cu}$ are observed, showing an inhomogeneous crystallization of nanowires. During the preparation of the samples for XRD analysis, it is possible to obtain some copper oxide (Figure 2(b)) due to the chemical removal of the Au layer on the bottom of the membrane. ${ }^{27}$ The higher content of $\mathrm{Cu}$ in $\mathrm{Ni}_{70} \mathrm{Cu}_{30}$ nanowires promotes a shift to smaller angle in the main [111] peak (Figure 2(c)). For intermediate $\mathrm{Cu}$ content (e.g., $45 \leq \mathrm{x} \leq 60$, see Figures 2(d) and 2(e)), we observe two [111] peaks partly overlapping that we ascribe to Ni-like and $\mathrm{Cu}$-like structures, respectively (note that the relative amplitude of the peaks changes with the $\mathrm{Cu}$ content). For the higher $\mathrm{Cu}$ content (e.g. $\mathrm{x}=75$, Figure 2(f)) only one peak is observed, ascribed to the $\mathrm{Cu}$-like structure. However, a shoulder is still detected in all the peaks as remains of the presence of Ni.

Figures 3(a) and 3(b) show a bright field TEM image and the SAED pattern of two representative nanowires with a selected intermediate composition of $\mathrm{Ni}_{55} \mathrm{Cu}_{45}$ and an average diameter of $60 \mathrm{~nm}$, which illustrate the microstructure of the nanowires. They present polycrystalline structure as demonstrated by the presence of diffraction rings indexed as (111), (200), and (220) sets of reflections of a cubic $f c c$ structure, in accordance with XRD data (Figure 2(d)). A detail of one of these nanowires is shown in the HRTEM image, where the good crystallinity of the nanowire is evidenced.

Figures 4-6 present the magnetic properties, coercivity $\left(H_{c}\right)$ and squareness $\left(M_{r} / M_{s}\right)$, of the nanowire arrays when the magnetic field is applied parallel $(\|)$ and perpendicular $(\perp)$ to the nanowires. The results are interpreted considering the crystal structure (magnetocrystalline anisotropy) and geometry of nanowires (shape anisotropy).

Figure 4 shows the coercivity and the squareness of the loops for $\mathrm{NiCu}$ wire arrays as function of the composition for $80 \mathrm{~nm}$ diameter and $8 \mu \mathrm{m}$ long nanowires arrays. For the perpendicular field configuration, both the squareness and coercivity increase continuously with $\mathrm{Cu}$ content. A different behaviour is observed in the parallel field configuration, where the coercivity and squareness show a relative maximum at around $45 \% \mathrm{Cu}$ content. With increasing the $\mathrm{Cu}$

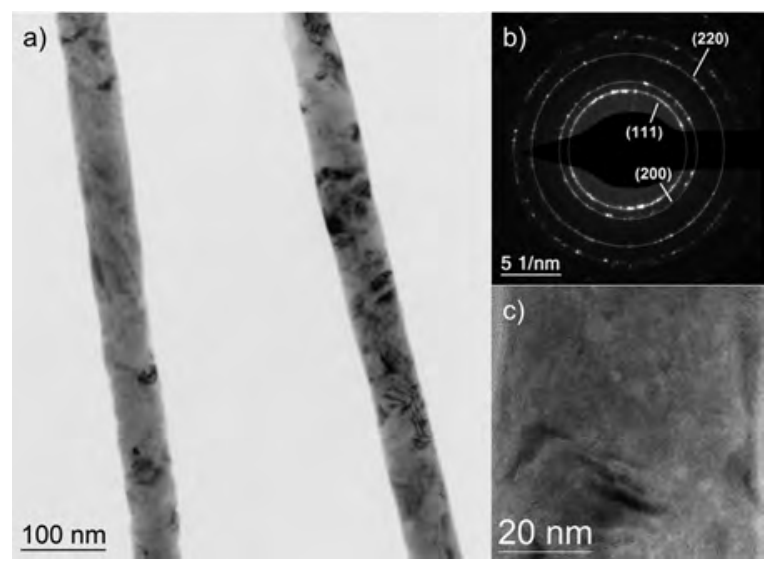

FIG. 3. Bright field TEM image of NiCu nanowires (a), SAED pattern showing the polycrystalline structure of the nanowires (b), and HRTEM image of one of the nanowires (c).

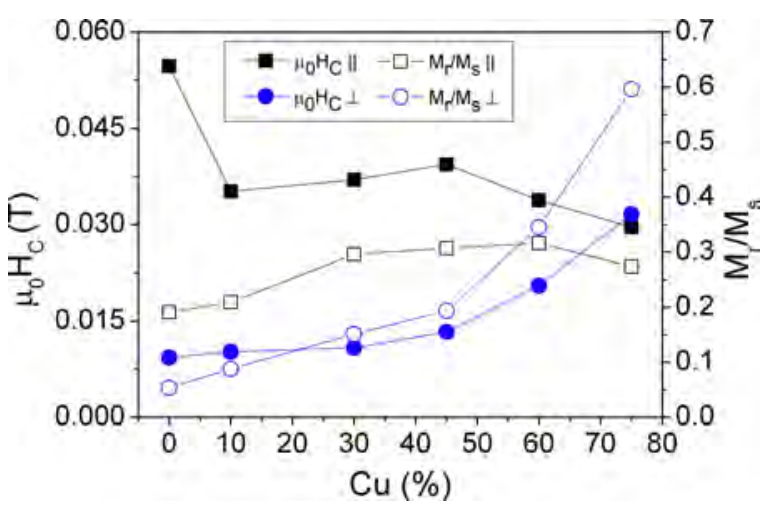

FIG. 4. Compositional dependence of coercive field and squareness of $80 \mathrm{~nm}$ diameter and $8 \mu \mathrm{m}$ long nanowire array.

content, apart from a decrease in the saturation magnetization (not shown here), we can deduce an overall evolution of the magnetization easy axis towards the perpendicular direction of the nanowires together with a likely modification in the magnetization reversal mechanism.

Magnetic properties of $\mathrm{NiCu}$ nanowires can be further tailored by tuning the geometrical parameters of nanowires such as length and diameter (Figure 5).

Figure 5(a) shows the nanowires length dependence of coercive field and squareness for $\mathrm{Ni}_{70} \mathrm{Cu}_{30}$ nanowires with diameter of $80 \mathrm{~nm}$. Both magnitudes, measured along the wires, increase with the length of the nanowires up to around $1 \mu \mathrm{m}$ (inset in Figure 5(a)) seemingly ascribed to an increase of the shape anisotropy. For longer nanowires (e.g., between
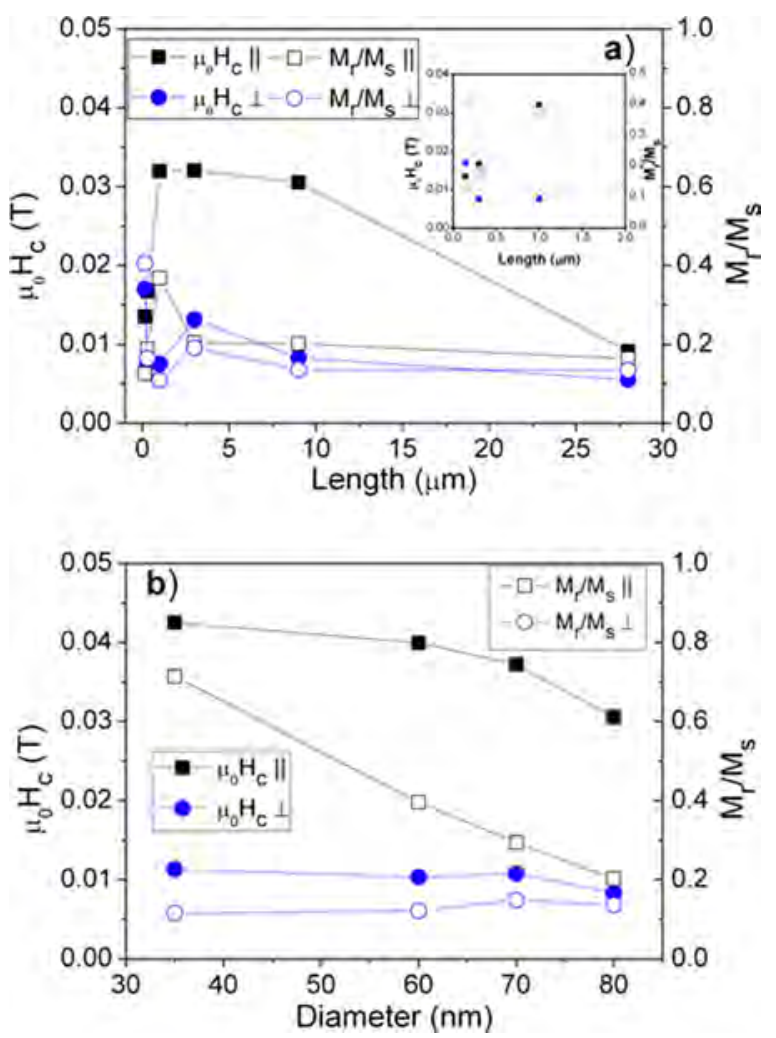

FIG. 5. Coercivity and squareness as a function of the length (for wires with diameter of $80 \mathrm{~nm}$ ) (a); and of the diameter (for wires with a length of $8 \mu \mathrm{m}$ ) (b) of $\mathrm{Ni}_{70} \mathrm{Cu}_{30}$ nanowires. 

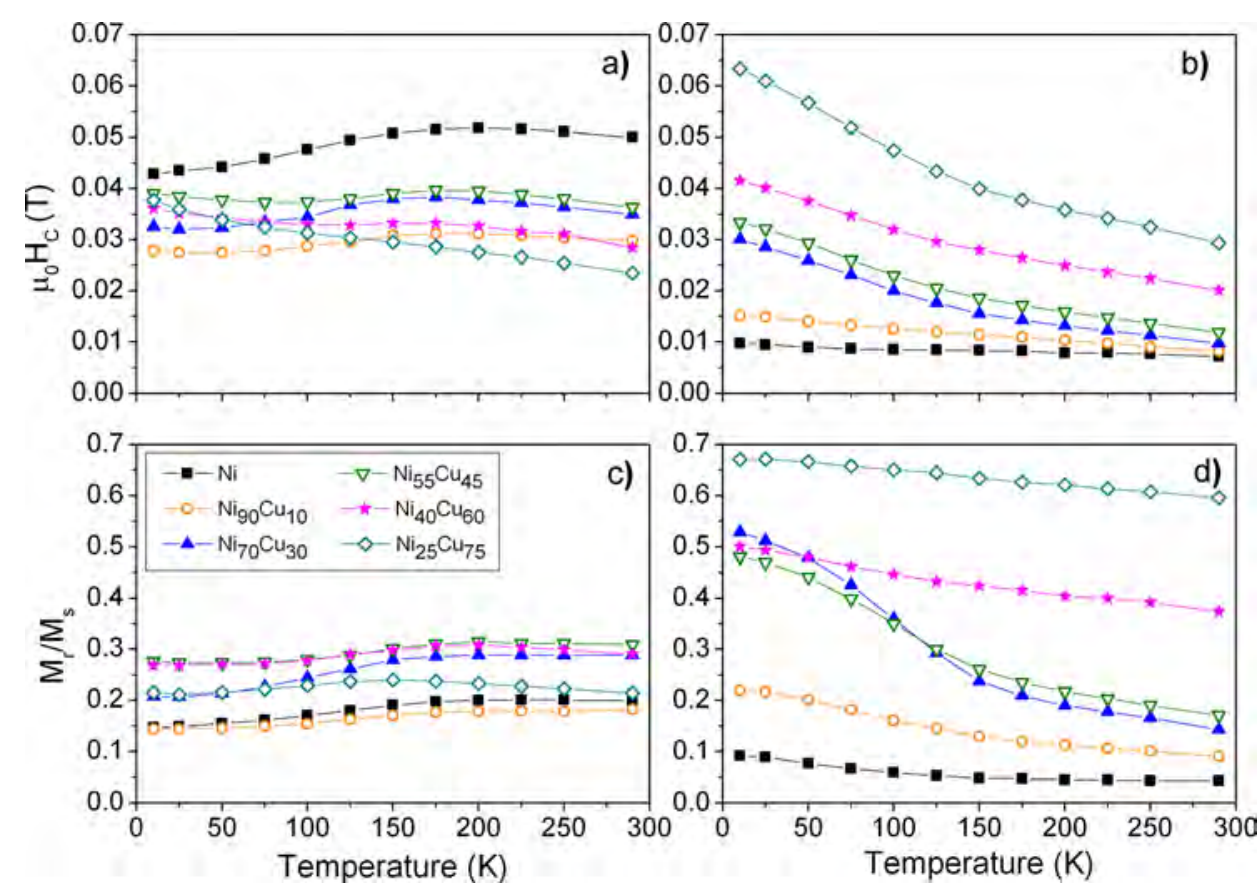

FIG. 6. Temperature dependence of coercivity and squareness of different composition $\mathrm{NiCu}$ nanowires $(80 \mathrm{~nm}$ diameter and $8 \mu \mathrm{m}$ length), when the magnetic field is applied parallel (a and $c$ ) and perpendicular (b and d) to the nanowires.
1 and $8 \mu \mathrm{m}$ ), these properties decrease slightly due to the magnetostatic interactions between neighbouring nanowires: the dipole interactions increase with nanowires length, giving a higher interaction field in the opposite direction. ${ }^{2,28}$ If their length is higher than $8 \mu \mathrm{m}$, both coercivity and squareness show a more pronounced decrease which can be attributed to enhanced magnetostatic interactions among wires $^{2,29}$ and to the averaging of inhomogeneities in morphology or in composition along the length of the nanowires due to the decrease of ions in the electrolyte during the electrodeposition. ${ }^{28,30,31}$

Figure 5(b) shows the dependence of coercivity and squareness as function of the nanowire diameters for $\mathrm{Ni}_{70} \mathrm{Cu}_{30}$ nanowires with length of $8 \mu \mathrm{m}$. The coercivity and squareness of nanowires decrease with their diameter. This is interpreted again considering an enhancement of the magnetostatic interactions among nanowires as they get closer to each other by increasing the diameter (the variation in diameter takes place for constant center-to-center distance).

In order to obtain additional information, magnetic characterization has been performed as a function of temperature. For this purpose, we have selected the particular case of nanowires with diameter of $80 \mathrm{~nm}$ and length of $8 \mu \mathrm{m}$. Figure 6 shows the temperature dependence of coercivity and squareness for a range of composition of nanowires $\mathrm{Ni}_{100-\mathrm{x}} \mathrm{Cu}_{\mathrm{x}}(0 \leq \mathrm{x} \leq 75)$ in the parallel and perpendicular field configurations. As observed, the variation in the composition of the nanowires has an overall stronger influence on the magnetic properties measured when the applied field is perpendicular to the nanowires than in parallel configuration.

Figure 6(a) shows the coercivity for the field applied parallel to the nanowires axis, $\mathrm{H}_{\mathrm{c}}(\|)$. For $\mathrm{x} \leq 30$, it increases with temperature until a maximum is reached at around 175-200 K and then it decreases at more elevated temperatures. For $45 \leq x \leq 60$, coercivity decreases with increasing temperature up to $100 \mathrm{~K}$, then it increases until a maximum is reached at about $175 \mathrm{~K}$, and decreases again. Finally, for $\mathrm{x}=75, \mathrm{H}_{\mathrm{c}}(\|)$ decreases continuously, almost linearly, with temperature.

Figure 6(c) shows that the behaviour of the squareness when the field is applied parallel to the nanowires, $M_{r} / M_{s}$ $(\|)$, is similar for all the composition. This magnitude increases until the temperature is around $175-200 \mathrm{~K}$, and it remains constant when $\mathrm{x}<60$. When the $\mathrm{Cu}$ content further increases, it is possible to observe a small decrease of $M_{r} / M_{s}$ (|l) with the temperature.

The coercivity for applied field perpendicular to the nanowires axis, $\mathrm{H}_{\mathrm{c}}(\perp)$, shows a very different behaviour (Figure 6(b)). By increasing the temperature, a pronounced decrease of coercivity is obtained, particularly for $x \geq 30$ when two different slopes can be observed. Note that the temperature where the two slopes intersect (around 175 K) coincides with the maximum of coercivity in Figure 6(a). A similar behaviour can be observed in Figure 6(d), where the squareness is represented as a function of perpendicular applied field.

In order to interpret the observed phenomenological behavior, we have to consider the different involved magnetic energy terms as well as the structural changes introduced by the increasing content in $\mathrm{Cu}$. The relevant energy contributions are the magnetocrystalline, $\mathrm{K}_{\text {crys }}$, the magnetoelastic, $\mathrm{K}_{\mathrm{elas}}$, and the shape, $\mathrm{K}_{\mathrm{sh}}$, energy density terms. On the other hand, as commented above, the XRD patterns suggest the presence of two [111] $f c c$ phases namely, a magnetic, Ni-like, and a non-magnetic, $\mathrm{Cu}$-like. In addition, from the modest and similar fractional reduction of the saturation magnetization with temperature for each composition (not shown), we deduce a similar Curie temperature for all the samples. That leads us to assume a low $\mathrm{Cu}$ content in the magnetic Ni-like phase, that is, increasing the $\mathrm{Cu}$ content gives rise to a reduction in magnetic moment of the nanowires while keeping similar Curie temperature. 
Consequently, we have first considered the evolution of the magnetic anisotropy terms of $\mathrm{Ni}$ to understand the observed behavior with temperature: (i) its magnetocrystalline anisotropy, $\mathrm{K}_{\text {crys }}$, is one order of magnitude higher at $10 \mathrm{~K}\left(\sim 5 \times 10^{4} \mathrm{~J} / \mathrm{m}^{3}\right)$ than at room temperature $\left(\sim 5 \times 10^{3} \mathrm{~J} /\right.$ $\mathrm{m}^{3}$ ); (ii) a significant magnetoelastic anisotropy, $\mathrm{K}_{\text {elas }}$, (perpendicular to the nanowires axis) appears at low temperature due to the different thermal expansion coefficient of $\mathrm{Ni}$ and alumina, ${ }^{2,32}$ (iii) the shape anisotropy, $\mathrm{K}_{\mathrm{sh}}$, can be assumed to be nearly constant owing to the reduced change in saturation magnetization in this temperature range.

On the other hand, we expect that increasing the $\mathrm{Cu}$ content gives rise to a reduction in the strength of all three energy terms. Note particularly that for high $\mathrm{Cu}$ content, the macroscopic (e.g., shape) anisotropy can be significantly altered by the structural changes induced by the dominant presence of $\mathrm{Cu}$-like phase.

For low content of $\mathrm{Cu}(\mathrm{x}<30)$, and at low temperatures, all the magnetic anisotropies would show similar values although $\mathrm{K}_{\mathrm{sh}}$ is the predominant. ${ }^{33}$ As the temperature increases, $K_{\text {elas }}$ would decrease and this brings the increase of the coercivity for fields parallel to the nanowires axis (Figure 6(a)). ${ }^{2,33}$ At a certain temperature (around 175 K) which corresponds to the maximum value of coercivity, $\mathrm{K}_{\text {crys }}$ and $\mathrm{K}_{\text {elas }}$ would balance and already become small compared with $\mathrm{K}_{\mathrm{sh}}$, that determines the behavior at higher temperature.

For the larger contents of $\mathrm{Cu}(45 \leq \mathrm{x} \leq 75)$, the XRD patterns show two phases. This means that magnetic Ni-like nanocrystals would be surrounded by $\mathrm{Cu}$-like ones and therefore $\mathrm{K}_{\mathrm{sh}}$ would significantly diminish with the $\mathrm{Cu}$ content. At low temperature, $\mathrm{K}_{\mathrm{sh}}$ would be no more the predominant term and $\mathrm{H}_{\mathrm{c}}(\|)$ decreases with increasing temperature until $\mathrm{K}_{\text {cryst }}$ and $\mathrm{K}_{\text {elas }}$ are negligible and $\mathrm{K}_{\mathrm{sh}}$ becomes again the most important anisotropy. For $\mathrm{Ni}_{25} \mathrm{Cu}_{75}$, the most predominant phase is the $\mathrm{Cu}$-like, $\mathrm{K}_{\mathrm{sh}}$ for the magnetic nanocrystals could be perpendicular to the nanowire axis and the maximum for $\mathrm{H}_{\mathrm{c}}(\|)$ disappears. The two different slopes in $\mathrm{H}_{\mathrm{c}}$ $(\perp)$ (Figure 6(b)) should be then related with the predominance of the different magnetic anisotropies, $\mathrm{K}_{\text {crys }}$ and $\mathrm{K}_{\text {elas }}$ for low and $\mathrm{K}_{\mathrm{sh}}$ for high temperatures, respectively.

In contrast to the bulk $\mathrm{NiCu}$ alloy, $\mathrm{NiCu}$ nanowires show a different behavior with the temperature. The dependence of Curie temperature, $\mathrm{T}_{\mathrm{c}}$, on the $\mathrm{Cu}$ percentage for $\mathrm{NiCu}$ bulk samples is well known: $\mathrm{T}_{\mathrm{c}}$ is around $300 \mathrm{~K}$ for $\mathrm{Ni}_{70} \mathrm{Cu}_{30}$ and goes to zero for $\mathrm{Ni}_{44} \mathrm{Cu}_{56}$, that is, the alloy should be paramagnetic for $\mathrm{Cu}$ content higher than $56 \%{ }^{34}$ For nanowires with $\mathrm{Cu}$ content above $45 \%$, the magnetic behavior shown in Figure 4 at room temperature can be explained from the XRD spectra (Figure 2): two phases appear, one $\mathrm{Cu}$-like (paramagnetic due to the $\mathrm{Ni}$ atoms) and one Ni-like, ferromagnetic and with magnetic properties close to those of $\mathrm{Ni}$ and therefore with similar $\mathrm{T}_{\mathrm{c}}$. For $\mathrm{Ni}_{70} \mathrm{Cu}_{30}$ nanowires, the XRD spectrum does not indicate any segregation of $\mathrm{Cu}$ (Figure 2(c)), so the magnetic response should be very low due to the proximity to its Curie temperature. It could be related with the high specific surface of the nanowires compared with the bulk $\mathrm{k}^{35}$ and/or with the special magnetic behavior of these alloys. ${ }^{36,37}$

\section{CONCLUSIONS}

Magnetic properties of $\mathrm{NiCu}$ nanowires can be tailored by tuning the composition and the geometrical parameters of nanowires such as length and diameter. The highest values of coercivity and squareness along the $\mathrm{NiCu}$ nanowires are obtained for a $\mathrm{Cu}$ content of $45 \%$, length of $1 \mu \mathrm{m}$ and diameter of $35 \mathrm{~nm}$. However for $\mathrm{Cu}$ content higher than $45 \%$ two different phases appear, Ni-rich and $\mathrm{Cu}$-rich. The magnetic behaviour with the temperature can be explained by the reduction in shape anisotropy with increasing $\mathrm{Cu}$ content, together with an increase of magnetocrystalline and magnetoelastic anisotropies at low temperatures.

\section{ACKNOWLEDGMENTS}

This work was supported by the Spanish Ministerio de Economia y Competitividad under Project MAT201020798-C05-01.

${ }^{1}$ C. Z. Wang, G. W. Meng, Q. Q. Fang, X. S. Peng, Y. W. Wang, Q. Fang, and L. D. Zhang, J. Phys. D: Appl. Phys. 35, 738 (2002).

${ }^{2}$ H. Schlörb, V. Haehnel, M. S. Khatri, A. Srivastav, A. Kumar, L. Schultz, and S. Fähler, Phys. Status Solidi B 247, 2364 (2010).

${ }^{3}$ E. Ferain and R. Legras, Nucl. Instrum. Methods Phys. Res. B 267, 1028 (2009).

${ }^{4}$ A. Fert and L. Piraux, J. Magn. Magn. Mater. 200, 338 (1999).

${ }^{5}$ H. Masuda and K. Fukuda, Science 268, 1466 (1995).

${ }^{6}$ A. Santos, L. Vojkuvka, J. Pallarés, J. Ferré-Borrull, and L. F. Marsal, Nanoscale Res. Lett. 4, 1021 (2009).

${ }^{7}$ M. E. Toimil-Molares, V. Buschmann, D. Dobrev, R. Neumann, R. Scholz, I. U. Schuchert, and J. Vener, Adv. Mater. 13, 62 (2001).

${ }^{8}$ K. Nielsch, F. Müller, A.-P. Li, and U. Gösele, Adv. Mater. 12, 582 (2000).

${ }^{9}$ K. R. Pirota, M. Knobel, M. Hernández-Vélez, K. Nielsch, and M. Vázquez, Handbook of Nanoscience and Nanotechnology (Oxford University Press, USA, 2010), p. 772.

${ }^{10}$ Z. H. Yang, Z. W. Li, L. Liu, and L. B. Kong, J. Magn. Magn. Mater. 323, 2674 (2011).

${ }^{11}$ M. Vázquez and L. G. Vivas, Phys. Status Solidi B 248, 2368 (2011).

${ }^{12}$ V. Vega, J. García, W. O. Rosa, L. G. Vivas, V. M. Prida, B. Hernando, and M. Vázquez, J. Nanosci. Nanotechnol. 12, 7501 (2012).

${ }^{13}$ A. Blonder, J. P. Meier, B. Boudin, and J.-Ph. Ansermet, Appl. Phys. Lett. 65, 3019 (1994).

${ }^{14}$ E. Tóth-Kádár, L. Péter, T. Becsei, J. Tóth, L. Pogány, T. Tarnóczi, P. Kamasa, I. Bakonyi, G. Láng, Á. Cziráki, and W. Schwarzacher, J. Electrochem. Soc. 147, 3311 (2000).

${ }^{15}$ R. R. Oberle, R. C. Cammarata, C. M. Su, and M. Wuttig, J. Mater. Res. 14, 3837 (1999).

${ }^{16}$ D. S. Lashmore and M. P. Dariel, J. Electrochem. Soc. 135, 1218 (1988).

${ }^{17}$ M. Chen, P. C. Searson, and C. L. Chien, J. Appl. Phys. 93, 8253 (2003).

${ }^{18}$ F. Béron, L.-P. Carignan, D. Ménard, and A. Yelon, IEEE Trans. Magn. 44, 2745 (2008).

${ }^{19}$ E. Matei, I. Enculescu, M. E. Toimil-Molares, A. Leca, C. Ghica, and V. Kuncser, J. Nanopart. Res. 15, 1863 (2013).

${ }^{20}$ K. Ounadjela, R. Ferré, L. Louail, J. M. George, J. L. Maurice, L. Piraux, and S. Dubois, J. Appl. Phys. 81, 5455 (1997).

${ }^{21}$ X. Xu and G. Zangari, J. Appl. Phys. 97, 10A306 (2005).

${ }^{22}$ R. S. Liu, S. C. Chang, P. Y. Chen, S. F. Hu, and C. Y. Huang, J. Chin. Chem. Soc. 57, 888 (2010).

${ }^{23}$ M. Ciureanu, F. Beron, L. Clime, P. Ciureanu, A. Yelon, T. A. Ovari, R. W. Cochrane, F. Normandin, and T. Veres, Electrochim. Acta 50, 4487 (2005).

${ }^{24}$ C. Bran, Yu. P. Ivanov, J. García, R. P. del Real, V. M. Prida, O. Chubykalo-Fesenko, and M. Vázquez, J. Appl. Phys. 114, 043908 (2013).

${ }^{25}$ J. M. Yang, Y. T. Hsieh, D. X. Zhuang, and I. W. Sun, Electrochem. Commun. 13, 1178 (2011).

${ }^{26}$ D. S. Xue, J. L. Fu, and H. G. Shi, J. Magn. Magn. Mater. 308, 1 (2007).

${ }^{27}$ G. Filipič and U. Cvelbar, Nanotechnology 23, 194001 (2012). 
${ }^{28}$ S. Aravamudhan, J. Singleton, P. A. Goddard, and S. Bhansali, J. Phys. D: Appl. Phys. 42, 115008 (2009).

${ }^{29}$ L. Clime, P. Ciureanu, and A. Yelon, J. Magn. Magn. Mater. 297, 60 (2006).

${ }^{30}$ I. Z. Rahman, K. M. Razeeb, Md. Kamruzzaman, and M. Serantoni, J. Mater. Process. Technol. 153, 811 (2004).

${ }^{31}$ J. Azevedo, C. T. Sousa, J. Ventura, A. Apolinario, A. Mendes, and J. P. Araujo, Mater. Res. Express 1, 015028 (2014).

${ }^{32}$ H. Zeng, S. Michalski, R. D. Kirby, D. J. Sellmyer, L. Menon, and S. Bandyopadhyay, J. Phys.: Condens. Matter 14, 715 (2002).
${ }^{33}$ A. Kumar, S. Fähler, H. Schlörb, K. Leistner, and L. Schultz, Phys. Rev. B 73, 064421 (2006).

${ }^{34}$ D. J. Chakrabarti, D. E. Laughlin, S. W. Chen, and Y. A. Chang, Phase Diagrams of Binary Nickel Alloys (ASM International, Materials Park, OH, 1991), p. 85.

${ }^{35}$ V. Leontyev, Phys. Status Solidi B 250, 103 (2013).

${ }^{36}$ T. J. Hicks, B. Rainford, J. S. Kouvel, G. G. Low, and J. B. Comly, Phys. Rev. Lett. 22, 531 (1969).

${ }^{37}$ R. W. Houghton, M. P. Sarachik, and J. S. Kouvel, Phys. Rev. Lett. 25, 238 (1970). 


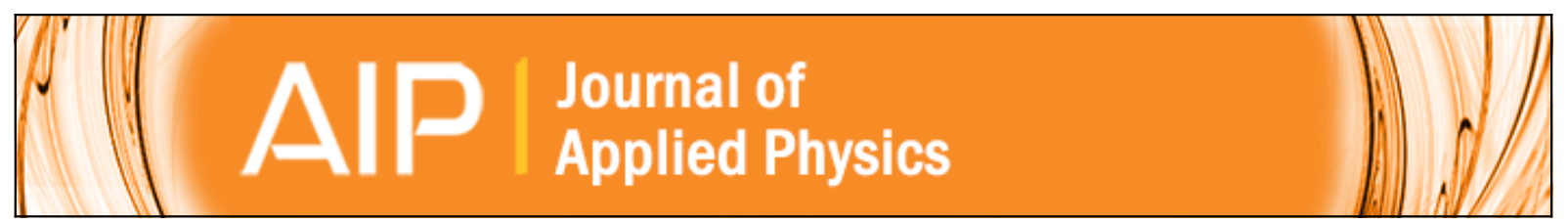

Magnetic behavior of NiCu nanowire arrays: Compositional, geometry and temperature dependence

E. M. Palmero, C. Bran, R. P. del Real, C. Magén, and M. Vázquez

Citation: Journal of Applied Physics 116, 033908 (2014); doi: 10.1063/1.4890358

View online: http://dx.doi.org/10.1063/1.4890358

View Table of Contents: http://scitation.aip.org/content/aip/journal/jap/116/3?ver=pdfcov

Published by the AIP Publishing

Articles you may be interested in

Gradient magnetic binary alloy nanowire

J. Appl. Phys. 115, 17B514 (2014); 10.1063/1.4864248

Structural and magnetic characterization of as-prepared and annealed FeCoCu nanowire arrays in ordered anodic aluminum oxide templates

J. Appl. Phys. 115, 133904 (2014); 10.1063/1.4870289

Fabrication of single crystalline, uniaxial single domain Co nanowire arrays with high coercivity

J. Appl. Phys. 115, 113902 (2014); 10.1063/1.4868582

Tuning the magnetization reversal process of $\mathrm{FeCoCu}$ nanowire arrays by thermal annealing

J. Appl. Phys. 114, 043908 (2013); 10.1063/1.4816479

Synthesis and magnetic properties of $\mathrm{Fe} 3 \mathrm{Pt}$ nanowire arrays fabricated by electrodeposition Appl. Phys. Lett. 86, 232506 (2005); 10.1063/1.1944210

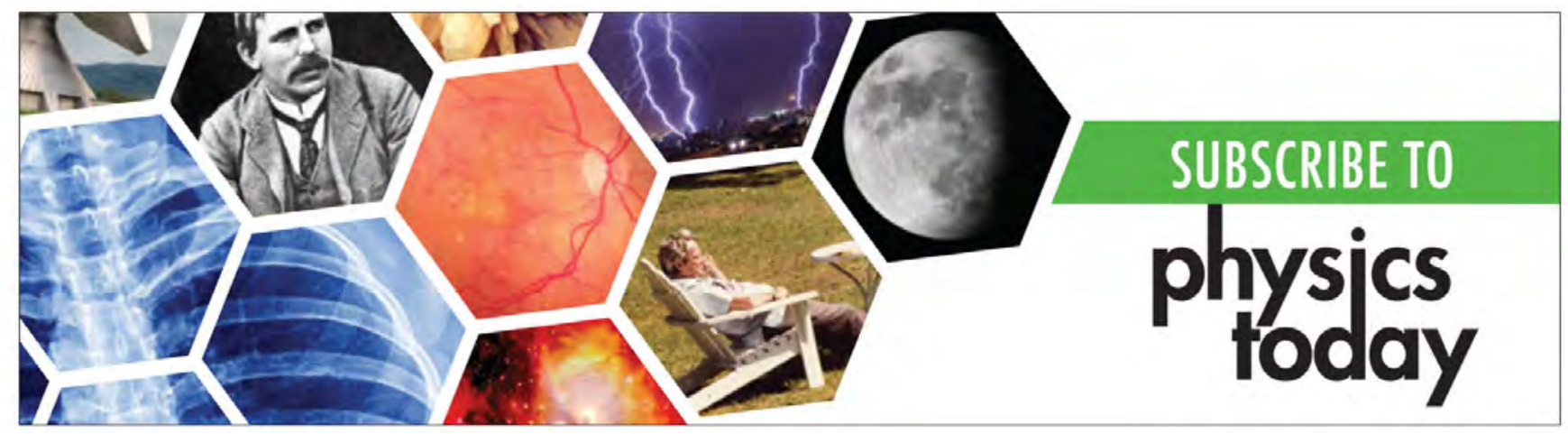

